\title{
Spinal cord dysfunction after COVID-19 infection
}

\author{
Gianluca Sampogna $^{1,2} \cdot$ Noemi Tessitore $^{1} \cdot$ Tatiana Bianconi $^{1} \cdot$ Alessandra Leo $^{1} \cdot$ Michele Zarbo $^{1}$ •

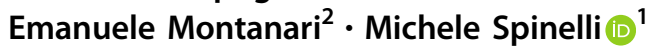

Received: 1 June 2020 / Revised: 14 September 2020 / Accepted: 15 September 2020

(C) International Spinal Cord Society 2020

\begin{abstract}
Introduction We observed individuals affected by spinal cord dysfunction (SCD) after coronavirus disease 2019 (COVID19). The aim of our report is to provide our initial experience with individuals experiencing SCD after COVID-19 in a referral center in Northern Italy, from February 21 to July 15, 2020.

Case presentation We report on three men with SCD after COVID-19. Case 1, aged 69 years, experienced T10 AIS B paraplegia upon awakening due to spinal cord ischemia from T8 to conus medullaris, besides diffuse thromboses, 27 days after the onset of COVID-19 symptoms. Case 2, aged 56 years, reported progressive cervicalgia 29 days after COVID-19 onset associated with C3 AIS C tetraplegia. Magnetic resonance imaging (MRI) revealed a C4-C6 spinal epidural abscess (SEA) requiring a C3-C4 left hemilaminectomy. Case 3, aged 48 years, reported backache together with lower limb muscle weakness on day 16 after being diagnosed with COVID-19. Exam revealed T2 AIS A paraplegia and an MRI showed a T1-T7 SEA. He underwent a T3-T4 laminectomy. Prior to SCD, all three individuals suffered from respiratory failure due to COVID-19, required mechanical ventilation, had cardiovascular risk factors, experienced lymphopenia, and received tocilizumab (TCZ).

Discussion To our knowledge, this is the first report of SCD after COVID-19. Based on our experience, we did not observe a direct viral infection, but there were two different etiologies. In Case 1, the individual developed spinal cord ischemia, whereas in Cases 2 and 3 SEAs were likely related to the use of TCZ used to treat COVID-19.
\end{abstract}

\section{Introduction}

On February 21, 2020, the outbreak of the coronavirus disease 2019 (COVID-19) started in Italy [1]. This infectious disease, caused by severe acute respiratory syndrome coronavirus 2 (SARS-CoV-2), represented a significant effort for our healthcare system [2].

In this scenario, our Unipolar Spinal Unit (USU) created a strict access protocol to maintain a COVID-19-free status [3]. Individuals with spinal cord dysfunction (SCD) are significantly frail, so COVID-19 may be associated with life-threatening presentations in this population $[4,5]$. Our

Michele Spinelli

michele.spinelli@ospedaleniguarda.it

1 Unipolar Spinal Unit and Neurourology Service, ASST Grande Ospedale Metropolitano Niguarda, Milan, Italy

2 Unit of Urology, Fondazione IRCCS Ca' Granda, Ospedale Maggiore Policlinico, Università degli Studi di Milano, Milan, Italy hospitalized patients could not receive visitors. Staff worked only in our USU, and doctors performed most outpatient visits with telemedicine. All our rules maintained a COVID19-free USU.

In the midst of these precautions, however, we admitted three individuals with SCD after COVID-19 from February 21 to July 15, 2020. All individuals had a positive nasopharyngeal swab (NPS) for SARS-CoV-2 infection prior to their admission. Although the last NPS had been negative when they entered our USU, we maintained these cases under isolation for 2 weeks as a further precaution. In all cases, the same author assessed the ASIA Impairment Scale (AIS) grade to evaluate the neurological level [6]. On July 15, 2020, all cases are still hospitalized in our USU.

The aim of our report is to provide our initial experience with people experiencing SCD after COVID-19 in a referral USU in the Northern Italian region most affected by the SARS-CoV-2 infection.

We conducted this study in accordance with the Declaration of Helsinki, and each individual provided written informed consent. 


\section{Case 1}

This 69-year-old male with arterial hypertension (HTN) and diabetes mellitus (disease burden estimated by the Charlson comorbidity index $(\mathrm{CCI})=3$ ) developed fever, cough, and anosmia [7]. Seven days later, he went to the emergency department (ED) due to respiratory failure. Initially, he required continuous positive airway pressure and after 5 days he underwent mechanical ventilation. His treatment included tocilizumab (TCZ), prophylactic low-molecularweight heparin (LMWH), hydroxychloroquine (HCQ), and lopinavir/ritonavir. Since he suffered from diabetes, his metformin was changed to insulin, but optimal glycemic control represented a significant challenge.

On day 25, he developed thromboses at the level of right posterior tibial vein, right small saphenous vein, right internal jugular and subclavian veins (central venous catheter-related thrombosis). In addition, the computed tomography scan showed an infarction of the left middle third posterior renal cortex and a left lower lobe pulmonary embolism. Blood tests revealed high levels of D-dimer $(>5000 \mathrm{ng} / \mathrm{mL})$ and an inferior vena cava filter was placed.

On day 27, the gentleman woke up with paraplegia. Magnetic resonance imaging (MRI) showed ischemia from T8 to the conus medullaris and the neurological level was T10 AIS B. This spectrum was coherent with the anterior spinal artery syndrome due to the occlusion of the artery of Adamkiewicz. When he entered our Unit, the spinal cord independence measure $(\mathrm{SCIM}$ ) was 12 (self-care $=4$; respiration and sphincter management $=8$; mobility $=0$ ) [8]. Later, he started clean intermittent catheterization (CIC). As for bowel management, he is receiving polyethylene glycol (PEG) and sennosides. He started to sit on the toilet. We are evaluating early transanal irrigation. After 60 days of rehabilitation, the SCIM raised up to 45 (self-care $=16$; respiration and sphincter management $=22$; mobility $=7$ ). Currently, he requires minimal assistance at a wheelchair level.

\section{Cases 2 and 3}

Case 2 is a 56-year-old male suffering from HTN, dyslipidemia, and trigeminal neuralgia $(\mathrm{CCI}=1)$. He first developed fever and cough, and, after 7 days, he suffered from respiratory failure, initially requiring noninvasive ventilation (NIV), followed by mechanical ventilation. Both NPS and bronchial aspirate, performed after tracheostomy, confirmed SARS-CoV-2 infection, and chest x-ray showed bilateral atypical pneumonia. He received TCZ, prophylactic LMWH, HCQ, and azithromycin. He was discharged home after 16 days.

Six-day post discharge (29 days after COVID-19 symptom onset), he experienced cervicalgia, right upper limb dysesthesia, and upper limb weakness. Since the pain was unresponsive to analgesics, he presented 4 days later to our ED where he underwent spine MRI, showing a C4-C6 spinal epidural abscess (SEA). In addition, he had fever up to $39.5^{\circ} \mathrm{C}$ and blood cultures revealed methicillinsusceptible Staphylococcus aureus (MSSA). He underwent a C3-C4 left hemilaminectomy. We observed neither intraoperative nor early postoperative complications. The intraoperative microbiological examination revealed MSSA. After surgery, neurologic exam assessed C3 AIS C tetraplegia. His clinical manifestation, characterized by a greater motor loss in the upper limbs than in the lower limbs, was consistent with central cord syndrome. He started CIC. As for bowel management, he is receiving PEG and sennosides. He started to sit on the toilet. When he was admitted, the SCIM was 10 (self-care $=0$; respiration and sphincter management $=8$; mobility $=2$ ). He has recently started to walk with a walking frame. After 60 days of rehabilitation, the SCIM was 73 (self-care $=14$; respiration and sphincter management $=33$; mobility $=26$ ).

Case 3 is a 48-year-old male with HTN and obesity (CCI $=0$ ) who developed fever and cough. After 7 days, his condition deteriorated, and he presented with respiratory failure, initially requiring NIV and, later, mechanical ventilation. We confirmed COVID-19 diagnosis with NPS and - after tracheostomy_bronchial aspirate. He received TCZ, high-dose corticosteroids, prophylactic LMWH, HCQ, lopinavir/ritonavir, and acyclovir.

On day 16, he reported backache associated with lower limb muscle weakness. After 4 days he underwent spine MRI, showing a T1-T7 SEA. The patient underwent a T3-T4 laminectomy. The intraoperative microbiological analyses revealed MSSA infection. On the 5th post operative day, the patient had fever, which was treated with a shift of antibiotic therapy. Blood cultures were positive for Enterococcus faecalis. After surgery, he was transferred to our USU, where the neurologic exam revealed a T2 AIS A paraplegia. The SCIM improved from 2 (self-care $=0$; respiration and sphincter management $=2$; mobility $=0$ ) to 47 (self-care $=14$; respiration and sphincter management $=$ 27; mobility $=6$ ) after 60 days of rehabilitation. We removed his tracheostomy, and he started CIC. As for bowel management, he is receiving PEG and sennosides, and has not started to sit on the toilet so far. He is using a corset and is not yet independent at a wheelchair level.

\section{Discussion}

Most individuals infected with COVID-19 experience mild symptoms, including fever, cough, sore throat, nasal congestion, diarrhea, dizziness, muscle weakness, anosmia, ageusia, and headache [9]. After 1 week, up to one-third of 
patients might present with respiratory failure, and $26 \%$ of them will require intensive care [10]. Several lifethreatening complications have been directly or indirectly associated with COVID-19, including venous thromboembolism, disseminated intravascular coagulation, cardiac injury, Kawasaki disease, and multiorgan failure [11-13].

SARS-CoV-2 has also been detected in the brain and cerebrospinal fluid. As a consequence of the neurotropic and neuro-invasive potential of this virus, it has been reported that $36.4 \%$ of patients with COVID-19 suffer from neurological complications, and up to $45.5 \%$ patients in case of severe SARS-CoV-2 infection [14]. Reported neurological manifestations have included dizziness, headache, impaired consciousness, acute cerebrovascular disease, ataxia, seizure, taste impairment, smell impairment, vision impairment, nerve pain, and Guillain-Barre syndrome $[15,16]$. The impact of COVID-19 on individuals with SCD has been reported. However, to the best of our knowledge, SCD after COVID-19 has not been described [17-22].

Case 1 presented a typical ischemic pattern of SCD: acute onset, low thoracic injury, and incomplete neurological level. This is in line with the well-known prothrombotic character of the SARS-CoV-2, confirmed by elevated D-dimer levels and hyperfibrinogemia, while abnormalities in prothrombin time, partial thromboplastin time, and platelet counts are initially uncommon [23].

SARS-CoV-2 has been associated with bacterial and fungal co-infections [24]. An infectious SCD etiology was detected in our other two cases: we observed SEAs due to MSSA, and a progressive clinical presentation consistent with an infectious etiology (they suffered from an escalating backache which was unresponsive to analgesics).

We believe that these abscesses may have been precipitated by the immunomodulatory therapy (IMT) we administered to treat SARS-CoV-2 infection. The treatment of COVID-19 is currently a matter of debate. During the initial period of the COVID-19 emergency, we experienced the release of weekly hospital protocols. Indeed, some drugs were not shared among the included cases. However, TCZ was a constant in $3 / 3$ cases [25]. TCZ is a humanized monoclonal antibody against the interleukin-6 receptor which proved to be effective against the cytokine storm induced by SARS-CoV-2. Following the initial success, this drug became freely available in Italy. Among TCZ potential adverse effects, abscesses in different body parts had been reported over the years [26, 27]. In general, IMT (e.g., corticosteroids, thiopurines, methotrexate, calcineurin inhibitors, and antitumor necrosis factor agents) may favor infections [28]. Therefore, the combination of COVID-19 and IMT might provoke abscesses, like the described SEAs.

A severe COVID-19 complication like SCD may be associated with specific risk factors. Patients with cardiovascular, pulmonary, and immunological diseases showed severe COVID-19 presentations. A recent metaanalysis outlined lymphopenia (lymphocyte count $<1500 / \mu \mathrm{L}$ ) was associated with nearly threefold increased risk of severe COVID-19 (random effects model, odds ratio $=2.99,95 \%$ CI: 1.31-6.82) [29]. When they were admitted to the ED, all our cases presented lymphopenia (Case $1=730 / \mu \mathrm{L}$; Case $2=540 / \mu \mathrm{L} ;$ Case $3=270 / \mu \mathrm{L}$ ), showing a significant risk factor for severe COVID-19, besides other predisposing comorbidities, like HTN, diabetes, and obesity.

Despite other viruses (e.g., human immunodeficiency virus, poliovirus, and arboviruses), we did not observe a SARS-CoV-2 infection of the spinal cord provoking a direct injury [30]. Indeed, our three cases developed SCD after COVID-19 for two different mechanisms: occlusion of the artery of Adamkiewicz and MSSA-related SEAs, probably precipitated by the IMT administered to treat COVID-19. This significant difference should be taken into account for preventing SCD while treating COVID-19.

Although the included sample is small, it represents a homogenous cohort admitted to our USU for rehabilitation [31]. Therefore, the population is representative of the incidence and prevalence of SCD associated with COVID19 by a referral USU in the Northern Italian region most affected by the SARS-CoV-2 diffusion.

Considering the impact of SCD on quality of life and national healthcare systems, we advocate the collection and sharing of data regarding individuals with SCD after COVID-19.

\section{Conclusions}

To our knowledge, this report is the first to describe new SCD after COVID-19. Our initial experience showed both ischemic and infectious etiology of SCD. Since COVID-19 complications are increasing, and they are often unfamiliar to most healthcare professionals, involved clinicians should be informed to face with this severe complication, addressing a prompt diagnosis and an efficacious treatment to decrease the SCD-related morbidity and public health burden.

Acknowledgements We thank Adriana Cassinis and Raffaella Tosi for their insights and helpful discussions, and all the staff working in our Unipolar Spinal Unit for their help in managing patients and finding medical records.

\section{Compliance with ethical standards}

Conflict of interest The authors declare that they have no conflict of interest.

Publisher's note Springer Nature remains neutral with regard to jurisdictional claims in published maps and institutional affiliations. 


\section{References}

1. Livingston E, Bucher K. Coronavirus disease 2019 (COVID-19) in Italy. JAMA. 2020;323:1335.

2. Zhu N, Zhang D, Wang W, Li X, Yang B, Song J, et al. A novel coronavirus from patients with pneumonia in China, 2019. N Engl J Med. 2020;382:727-33.

3. Bartolo M, Intiso D, Lentino C, Sandrini G, Paolucci S, Zampolini M. Urgent measures for the containment of the coronavirus (Covid-19) epidemic in the neurorehabilitation/rehabilitation departments in the phase of maximum expansion of the epidemic. Front Neurol. 2020;11:423.

4. Agosti E, Giorgianni A, Locatelli D. Impact of COVID-19 outbreak on spinal pathology: single center first impression. Spinal Cord. 2020. https://doi.org/10.1038/s41393-020-0480-0.

5. Dicks MA, Clements ND, Gibbons CR, Verduzco-Gutierrez M, Trbovich M. Atypical presentation of Covid-19 in persons with spinal cord injury. Spinal Cord Ser Cases. 2020;6:38.

6. Betz R, Biering-Sørensen F, Burns SP, Donovan W, Graves DE, Guest J, et al. The 2019 revision of the International Standards for Neurological Classification of Spinal Cord Injury (ISNCSCI)— what's new? Spinal Cord. 2019;57:815-7.

7. Quan H, Li B, Couris CM, Fushimi K, Graham P, Hider P, et al. Updating and validating the charlson comorbidity index and score for risk adjustment in hospital discharge abstracts using data from 6 countries. Am J Epidemiol. 2011;173: 676-82.

8. Catz A, Itzkovich M, Steinberg F, Philo O, Ring H, Ronen J, et al. The Catz-Itzkovich SCIM: a revised version of the spinal cord independence measure. Disabil Rehabil. 2001;23:263-8.

9. Chang T-H, Wu J-L, Chang L-Y. Clinical characteristics and diagnostic challenges of pediatric COVID-19: a systematic review and meta-analysis. J Formos Med Assoc. 2020;119:982-9.

10. Wang D, Hu B, Hu C, Zhu F, Liu X, Zhang J, et al. Clinical characteristics of 138 hospitalized patients with 2019 novel coronavirus-infected pneumonia in Wuhan, China. JAMA. 2020;323:1061-9.

11. Jones VG, Mills M, Suarez D, Hogan CA, Yeh D, Bradley Segal J, et al. COVID-19 and Kawasaki disease: novel virus and novel case. Hosp Pediatr. 2020;10:537-40.

12. Bikdeli B, Madhavan MV, Jimenez D, Chuich T, Dreyfus I, Driggin E, et al. COVID-19 and thrombotic or thromboembolic disease: implications for prevention, antithrombotic therapy, and follow-up. J Am Coll Cardiol. 2020;75:2950-73.

13. Connors JM, Levy JH. COVID-19 and its implications for thrombosis and anticoagulation. Blood. 2020;135:2033-40.

14. Mao L, Jin H, Wang M, Hu Y, Chen S, He Q, et al. Neurologic manifestations of hospitalized patients with coronavirus disease 2019 in Wuhan, China. JAMA Neurol. 2020;77:1-9.

15. Helms J, Kremer S, Merdji H, Clere-Jehl R, Schenck M, Kummerlen $\mathrm{C}$, et al. Neurologic features in severe SARS-CoV-2 infection. N Engl J Med. 2020;382:2268-70.
16. Toscano G, Palmerini F, Ravaglia S, Ruiz L, Invernizzi P, Cuzzoni MG, et al. Guillain-Barré syndrome associated with SARSCoV-2. N Engl J Med. 2020;382:2574-6.

17. Palipana D. COVID-19 and spinal cord injuries: the viewpoint from an emergency department resident with quadriplegia. Emerg Med Australas. 2020. https://doi.org/10.1111/1742-6723.13525.

18. Righi G, Del Popolo G. COVID-19 tsunami: the first case of a spinal cord injury patient in Italy. Spinal Cord Ser Cases. 2020;6:22.

19. Stillman MD, Capron M, Alexander M, Di Giusto ML, Scivoletto G. COVID-19 and spinal cord injury and disease: results of an international survey. Spinal Cord Ser Cases. 2020;6:21.

20. Korupolu R, Stampas A, Gibbons C, Hernandez Jimenez I, Skelton F, Verduzco-Gutierrez M. COVID-19: screening and triage challenges in people with disability due to spinal cord injury. Spinal Cord Ser Cases. 2020;6:35.

21. Alexander M. Let's conquer COVID-19 and sustain our abilities. Spinal Cord Ser Cases. 2020;6:19.

22. Rodríguez-Cola M, Jiménez-Velasco I, Gutiérrez-Henares F, López-Dolado E, Gambarrutta-Malfatti C, Vargas-Baquero E, et al. Clinical features of coronavirus disease 2019 (COVID-19) in a cohort of patients with disability due to spinal cord injury. Spinal Cord Ser Cases. 2020;6:39.

23. Ranucci M, Ballotta A, Di Dedda U, Bayshnikova E, Dei Poli M, Resta M, et al. The procoagulant pattern of patients with COVID19 acute respiratory distress syndrome. J Thromb Haemost. 2020;18:1747-51.

24. Rawson TM, Moore LSP, Zhu N, Ranganathan N, Skolimowska $\mathrm{K}$, Gilchrist $\mathrm{M}$, et al. Bacterial and fungal co-infection in individuals with coronavirus: a rapid review to support COVID-19 antimicrobial prescribing. Clin Infect Dis. 2020:ciaa530. https:// doi.org/10.1093/cid/ciaa530.

25. Fu B, Xu X, Wei $\mathrm{H}$. Why tocilizumab could be an effective treatment for severe COVID-19? J Transl Med. 2020;18:1-5.

26. Fujita S, Kosaka N, Mito T, Hayashi H, Morita Y. Development of aseptic subcutaneous abscess after tocilizumab therapy in a patient with SAPHO syndrome complicated by amyloid A amyloidosis. Int J Rheum Dis. 2015;18:476-9.

27. Sakane H, Kaneko T, Yonemoto Y, Okamura K, Okura C, Suto T, et al. Ankle abscess under the use of tocilizumab in rheumatoid arthritis; a case report. Clin Rheumatol Relat Res. 2018;30:120-5.

28. Orlicka K, Barnes E, Culver EL. Prevention of infection caused by immunosuppressive drugs in gastroenterology. Ther Adv Chronic Dis. 2013;4:167-85.

29. Zhao Q, Meng M, Kumar R, Wu Y, Huang J, Deng Y, et al. Lymphopenia is associated with severe coronavirus disease 2019 (COVID-19) infections: a systemic review and meta-analysis. Int J Infect Dis. 2020;96:131-5.

30. New PW. A narrative review of pediatric nontraumatic spinal cord dysfunction. Top Spinal Cord Inj Rehabil. 2019;25:112-20.

31. López-Dolado E, Gil-Agudo A. Lessons learned from the coronavirus disease 2019 (Covid-19) outbreak in a monographic center for spinal cord injury. Spinal Cord. 2020;58:517-9. 